\title{
Development and validation of an oxygen dissociation assay, a screening platform for discovering, and characterizing hemoglobin- oxygen affinity modifiers
}

This article was published in the following Dove Press journal:

Drug Design, Development and Therapy

\author{
Mira P Patel' \\ Vincent Siu' \\ Abel Silva-Garcia' \\ Qing $X u^{2}$ \\ Zhe $\mathrm{Li}^{2}$ \\ Donna Oksenberg' \\ 'Biology Department, Global \\ Blood Therapeutics Inc., South San \\ Francisco, CA, USA; ${ }^{2}$ Chemistry \\ Department, Global Blood \\ Therapeutics Inc., South San \\ Francisco, CA, USA
}

\begin{abstract}
Introduction: Hemoglobin $(\mathrm{Hb})$ is a critical molecule necessary for all vertebrates to maintain aerobic metabolism. $\mathrm{Hb}$-oxygen $\left(\mathrm{O}_{2}\right)$ affinity modifiers have been studied to address various diseases including sickle cell disease, hypoxemia, tumor hypoxia, and wound healing. However, drug development of exogenous $\mathrm{Hb}$ modifiers has been hindered by the lack of a technique to rapidly screen compounds for their ability to alter $\mathrm{Hb}-\mathrm{O}_{2}$ affinity. We have developed a novel screening assay based upon the spectral changes observed during $\mathrm{Hb}$ deoxygenation and termed it the oxygen dissociation assay (ODA).
\end{abstract}

Methodology: ODA allows for the quantitation of oxygenated $\mathrm{Hb}$ at given time points during $\mathrm{Hb}$ deoxygenation on a 96-well plate. This assay was validated by comparing the ability of $500 \mathrm{Hb}$ modifiers to alter the $\mathrm{Hb}-\mathrm{O}_{2}$ affinity in the ODA vs the oxygen equilibrium curves obtained using the industry standard Hemox Analyzer instrument.

Results: A correlation $\left(R^{2}\right)$ of 0.7 indicated that the ODA has the potential to screen and identify potent exogenous $\mathrm{Hb}$ modifiers. In addition, it allows for concurrent comparison of compounds, concentrations, buffers, or $\mathrm{pHs}$ on the level of $\mathrm{Hb}$ oxygenation.

Conclusion: With a cost-effective, simple, rapid, and highly adaptable assay, the ODA will allow researchers to rapidly characterize $\mathrm{Hb}-\mathrm{O}_{2}$ affinity modifiers.

Keywords: hemox analyzer, in vitro assays, small-molecule screening, voxelotor

\section{Introduction}

Hemoglobin $(\mathrm{Hb})$ is a critical protein involved in binding, transporting, and off-loading oxygen. ${ }^{1-3}$ Due to this multifaceted role, $\mathrm{Hb}$ has evolved to exist in equilibrium between the liganded, oxygenated $\mathrm{Hb}$ (oxy $\mathrm{Hb}$ ) form and unliganded, deoxygenated (deoxy $\mathrm{Hb}$ ) form. ${ }^{1} \mathrm{Hb}$ is sensitive to allosteric modifiers, such as carbon dioxide $\left(\mathrm{CO}_{2}\right)$, hydrogen ions, and 2,3 diphosphoglycerate (2,3-DPG), which enables adequate binding, transport, and delivery of oxygen. ${ }^{1,4}$ Such allosteric modifiers enable $\mathrm{Hb}$ variable oxygen affinity in vivo. ${ }^{1,2}$ Modulating the oxygen $\left(\mathrm{O}_{2}\right)$ affinity of $\mathrm{Hb}$ with exogenous modifiers may be a promising approach for treating a range of diseases. ${ }^{1}$ For example, maintaining a portion of $\mathrm{Hb}$ in the high-affinity oxygenated state is beneficial for sickle cell disease (SCD), as it will delay the polymerization of deoxygenated sickle $\mathrm{Hb}(\mathrm{HbS})$, which is the major driver of the pathophysiology of the disease. ${ }^{5,6}$ In addition, increasing the $\mathrm{Hb}-\mathrm{O}_{2}$ affinity may benefit hypoxemia related to pulmonary diseases as it will increase $\mathrm{O}_{2}$ saturation in the lungs. ${ }^{7,8}$ Alternatively, decreasing $\mathrm{Hb}-\mathrm{O}_{2}$ affinity could be useful in diseases such as tumor hypoxia, wound healing, or ischemia where increased
Correspondence: Mira P Patel Biology Department, Global Blood Therapeutics, I7I Oyster Point Blvd., Suite 300, South San Francisco, CA 94080, USA

Tel +I 6507417712

Email mpatel@gbt.com 
$\mathrm{O}_{2}$ delivery to tissues is needed. ${ }^{1}$ For these reasons, various $\mathrm{Hb}$ modifiers have been identified that either increase (5-HMF, tucaresol, BW12C, voxelotor [previously known as GBT440], GBT1118) or decrease (RSR-13, ITPP) $\mathrm{Hb}-\mathrm{O}_{2}$ affinity. $5,7,9$

Drug discovery in the $\mathrm{Hb}-\mathrm{O}_{2}$ affinity modifier field has been hampered by limitations wherein instruments requiring high concentrations of $\mathrm{Hb}$ necessitate high concentrations of compounds for testing. $\mathrm{Hb}-\mathrm{O}_{2}$ affinity modifiers have been identified using clinical instrumentation that measures the partial pressure of $\mathrm{O}_{2}$ at which $\mathrm{Hb}$ is $50 \%$ saturated with $\mathrm{O}_{2}$ (p50) through oxygen equilibrium curves (OECs). ${ }^{10}$ These instruments have included tonometers, Hem-O-Scans, and TCS Hemox Analyzers. ${ }^{10}$ Though the TCS Hemox Analyzer and similar equipment are the standard in the field for characterizing $\mathrm{Hb}$ mutations with altered $\mathrm{Hb}-\mathrm{O}_{2}$ affinity, such instruments are not conducive to screening, or to performing structure-activity relationship (SAR)-based drug discovery, which often requires high-throughput screening. The TCS Hemox Analyzer, which is the most advanced instrumentation currently deployed, measures the oxygen partial pressure and concurrently measures $\mathrm{Hb}$ saturation with a Clark oxygen electrode and spectrophotometry, respectively. The TCS Hemox Analyzer uses a 20-30 $\mu \mathrm{M}$ Hb solution and a $5 \mathrm{~mL}$ cuvette, which prevents simultaneous measurements. Due to these limitations, measuring an OEC using the TCS Hemox Analyzer requires a minimum of 20 minutes per sample and sufficient compound to be tested against $20-30 \mu \mathrm{M}$ of $\mathrm{Hb}$ in a $5 \mathrm{~mL}$ cuvette. ${ }^{11}$ In addition, to measure reliable OECs, the compounds need to be soluble at the concentrations being tested. These factors are limiting when using OECs for drug discovery.

To overcome these drawbacks, researchers have attempted to develop a variety of miniaturization techniques. One such assay, the $\mathrm{Hb}$-oxygen binding assay, was developed by Nakagawa et al (2014) wherein changes were observed over a 14-hour period at three different $\mathrm{FO}_{2}$ (fraction of oxygen gas in chamber) levels in a 384-plate format. ${ }^{12}$ Based upon similar principles, we developed the oxygen dissociation assay (ODA), which uses the Soret (400-450 nm) and Q (500-600 nm) bands of $\mathrm{Hb}$ to measure the level of oxy $\mathrm{Hb}$ during deoxygenation within a 96-well plate format. ${ }^{5}$ In addition, the ODA was optimized for $\mathrm{Hb}$ tetramer concentrations as low as $3 \mu \mathrm{M}$, which consequently lowers the concentration of compounds utilized in screening. The design of the ODA will allow researchers to rapidly compare and characterize putative $\mathrm{Hb}-\mathrm{O}_{2}$ affinity modifiers across different concentrations, buffers, and $\mathrm{pH}$ conditions.

\section{Materials and methods Compounds}

Phytic acid (also known as inositol hexaphosphate) was purchased from MilliporeSigma, Burlington, MA, USA and efaproxiral was purchased from BePharm Ltd, Shanghai, China. All other compounds including reference compounds, tucaresol, voxelotor (formerly known as GBT440), and GBT1118, were synthesized at Global Blood Therapeutics, South San Francisco, CA, USA. ${ }^{5,7,13}$ For all studies herein, compounds were solubilized in $100 \%$ dimethyl sulfoxide (DMSO) at concentrations ranging from 10 to $100 \mathrm{mM}$.

\section{Buffers}

TES buffer, also known as TES/saline buffer, is $30 \mathrm{mM}$ TES (2-[[1,3-dihydroxy-2-(hydroxymethyl)pro-pan-2-yl]amino] ethanesulfonic acid), $140 \mathrm{mM}$ saline, $\mathrm{pH} 7.4$ at $25^{\circ} \mathrm{C}$ (unless noted otherwise). Phosphate buffer is $50 \mathrm{mM} \mathrm{KH}_{2} \mathrm{PO}_{4}$, $30 \mu \mathrm{M}$ 2,3-DPG, pH 7.4 (unless noted otherwise).

\section{Blood source}

Blood was either obtained through the Children's Hospital Oakland Research Institute (CHRCO, Oakland, CA, USA [Institutional Review Board \{IRB\} 2013-006]) or was purchased from the Stanford Blood Center (Stanford, CA, USA) under their approved protocols and with donor written informed consents.

\section{$\mathrm{Hb}$ purification}

Unique donors $(>30)$ were used for the studies presented herein. $\mathrm{Hb}$ was purified from individual donor red blood cells (RBCs) by gel filtration and DE-52 anion exchange chromatography. ${ }^{14}$ Samples of the eluted fractions were run on a Tris-glycine $12 \%$ acrylamide native gel where they were separated according to their isoelectric point (pI) allowing for their identification.

\section{Oxygen dissociation assay}

The ODA uses the spectral signatures of $\mathrm{Hb}$ (Soret bands: 400-450 nm and Q bands: 500-600 nm) to measure the level of oxy $\mathrm{Hb}$ during deoxygenation. Purified $\mathrm{Hb}$ ( $3 \mu \mathrm{M}$ tetramer in various buffers) $)^{15,16}$ was incubated for 1 hour under ambient air at $37^{\circ} \mathrm{C}$ in the presence or absence of various compounds in sealed 96-well, half-area, optically transparent polystyrene plates $\left(\mu\right.$ Clear $^{\circledR}$ bottom; Greiner Bio-One International $\mathrm{GmbH}$, Kremsmünster, Austria). After incubation, the plate seal was removed, and the samples were deoxygenated with gaseous dry $\mathrm{N}_{2}$ for 2 hours at $37^{\circ} \mathrm{C}$ in a SPECTROstar Nano (BMG Labtech, Inc., Ortenberg, Germany), an ultraviolet/visible (UV/Vis) absorbance spectrometer capable of reading full 
spectra in $<1$ second/well. Deoxygenation was achieved by blowing $\mathrm{N}_{2}$ over (but not directly into) the samples at $20 \mathrm{~L} / \mathrm{min}$. As the buffer and $\mathrm{Hb}$ equilibrate during deoxygenation, spectral measurements (350-700 nm, with a spectral resolution of $1 \mathrm{~nm}$ ) were obtained every 6 minutes to determine the oxy $\mathrm{Hb}$ level over time. In between each measurement, the plate was shaken in a figure 8 for 1 minute at $300 \mathrm{rpm}$. Data analysis was performed using Excel's LINEST function on the wavelengths ranging from 380 to $700 \mathrm{~nm}$. The $\mathrm{Hb}$ spectrum at time zero was used as the reference for $100 \%$ oxy $\mathrm{Hb}$, while the average spectra of $\mathrm{Hb}$ with $15 \mathrm{mM}$ sodium dithionite (Merck Millipore, Burlington, MA, USA), a reducing agent, was used as the reference for $100 \%$ deoxy $\mathrm{Hb}$ (spectra not shown). The results were then expressed as \%oxy $\mathrm{Hb} .{ }^{5}$ For the data presented within this manuscript, the issue of methemoglobin was resolved by a visual inspection of all spectra.

\section{Oxygen equilibrium curves}

To measure changes in the binding affinity of $\mathrm{O}_{2}$ to $\mathrm{Hb}, \mathrm{OECs}$ were collected using a TCS Hemox Analyzer (TCS Scientific Corporation, New Hope, PA, USA).${ }^{11} \mathrm{Hb}(25 \mu \mathrm{M}$ tetramer) was incubated for 45 minutes at $37^{\circ} \mathrm{C}$ with various concentrations of compounds in TES buffer ${ }^{3}$. Purified $\mathrm{Hb}$ samples were then oxygenated with compressed air for 10 minutes within the Hemox Analyzer. After oxygenation, the samples were deoxygenated with compressed $\mathrm{N}_{2}$ and OECs were collected during deoxygenation as previously described. ${ }^{11}$ From the OECs, p50 values were derived using TCS Hemox Analysis Software.

\section{$\mathrm{pH}$-dependent $\mathrm{Hb}-\mathrm{O}_{2}$ affinity}

For the $\mathrm{pH}$-dependent experiments, the ODA was adapted to allow comparison of various $\mathrm{pHs}$ within a single plate. In these studies, different pHs (6.8-8.0) were used in the columns of the 96 -well plates. Columns $1-3$ contained pH 6.8 buffer, columns 4-6 contained $\mathrm{pH} 7.0$ buffer, columns 7-9 had pH 7.4 buffer, and columns 10-12 had pH 8.0 buffer. Row $\mathrm{H}$ was the $\mathrm{Hb}$ control specific to each $\mathrm{pH}$ condition. $\mathrm{Hb}$ and compounds were incubated for 1 hour at $37^{\circ} \mathrm{C}$ under the four $\mathrm{pH}$ conditions used. After incubation, data collection and data analysis proceeded as in the standard ODA.

\section{$\mathrm{Hb}$ /human serum albumin (HSA) competition experiment}

For these studies, all work was conducted using the basic ODA method, with a minor adaptation to include a preincubation step of voxelotor or tucaresol with HSA (MilliporeSigma, Burlington, MA, USA, globulin and fatty acid free). The experiments were conducted using $50 \mathrm{mM}$

potassium phosphate buffer at $\mathrm{pH}$ 7.4. Voxelotor or tucaresol was diluted from a $10 \mathrm{mM}$ stock solution (in 100\% DMSO). HSA (at concentrations ranging from 0.02 to $200 \mu \mathrm{M}$ ) was incubated with $6 \mu \mathrm{M}$ voxelotor or $6 \mu \mathrm{M}$ tucaresol for 1 hour at room temperature. The samples were then diluted twofold with $\mathrm{Hb}$ and 2,3-DPG to a final concentration of 0.01-100 $\mu \mathrm{M}$ HSA, $30 \mu \mathrm{M}$ 2,3-DPG, $3 \mu \mathrm{M}$ voxelotor or tucaresol, and $3 \mu \mathrm{M} \mathrm{Hb}$ tetramer. These samples were then sealed and incubated at $37^{\circ} \mathrm{C}$ for an additional hour in a humidified incubator. Following the incubation, data collection and data analysis proceeded as in the standard ODA. As the maximal activity for tucaresol and voxelotor is not equivalent, the data were normalized to the maximal \%oxy $\mathrm{Hb}$ value. This normalization allowed for discerning the contributions of HSA binding for different compounds.

\section{Results Assay design}

All instruments used to determine the oxygen affinity of $\mathrm{Hb}$ rely on the spectral difference between oxy $\mathrm{Hb}$ and deoxy $\mathrm{Hb}$. The first step in validating the ODA was to monitor the spectral change from oxy $\mathrm{Hb}$ to deoxy $\mathrm{Hb}$ during the 2-hour deoxygenation process. After samples are incubated with $\mathrm{Hb}$ in 96-well half-area plates, they are placed in the spectrophotometer. The spectrophotometer is set up with a gas inlet that allows for the reading chamber to be filled with $\mathrm{N}_{2}$. When using a BMG SPECTROstar Nano, the microplate chamber can be filled with $\mathrm{N}_{2}$ directly (Figure 1A). As the chamber

A

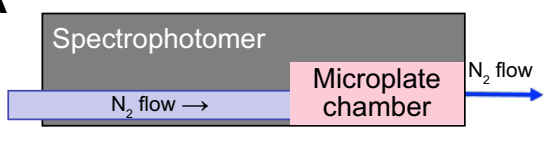

B At room air

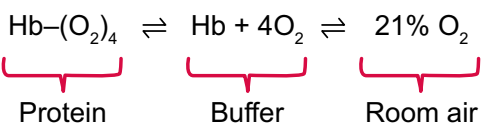

During deoxygenation

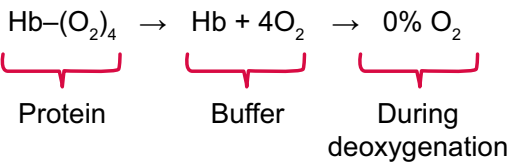

Figure I Schematics and principles of the oxygen dissociation assay.

Notes: (A) The flow of $\mathrm{N}_{2}$ within the spectrophotometer for the ODA. (B) The equilibrium for liganded $\mathrm{Hb}$ at room air and how it changes during the 2-hour deoxygenation. (C) While $\mathrm{N}_{2}$ is flowing across the top of each well, the sample deoxygenation is monitored by $\mathrm{Hb}$ spectra changes that are captured in an orthogonal direction to the gas flow.

Abbreviations: $\mathrm{Hb}$, hemoglobin; ODA, oxygen dissociation assay. 
fills, each well within the 96-well plate is exposed to diminishing levels of $\mathrm{O}_{2}$ (Figure 1B). This change in $\mathrm{O}_{2}$ levels alters the equilibrium between the well and the local atmosphere driving $\mathrm{O}_{2}$ out of the buffer. As oxy $\mathrm{Hb}$ releases $\mathrm{O}_{2}$ to the buffer, a spectral shift can be measured (Figure 1C). Humid ( $\geq 56 \%$ humidity) vs dry $\mathrm{N}_{2}$ was evaluated during assay development and it was found there was no quantitative difference between the two in terms of dehydration or deoxygenation (data not shown). Humidity was measured using a hygrometer placed up against the vent port in the spectrophotometer.

Figure 2A shows the spectral measurements of $\mathrm{Hb}$ at the beginning and end of the 2-hour deoxygenation process. The characteristic Soret peak at $415 \mathrm{~nm}$ transitions toward $430 \mathrm{~nm}$ (the $\lambda_{\max }$ of deoxy $\mathrm{Hb}$ ). Similarly, in the Q-band region, the double peaks of oxy $\mathrm{Hb}(541,577 \mathrm{~nm})$ transition toward a single peak at $555 \mathrm{~nm}$, which is also characteristic of deoxy $\mathrm{Hb}$. Data analysis was performed using Excel's LINEST function on the wavelengths ranging from 380 to $700 \mathrm{~nm}$, as described in the Materials and methods. The results were then expressed as \%oxy $\mathrm{Hb}$. The above analysis was conducted on the two spectra from Figure $2 \mathrm{~A}$ as well as the 18 spectra in between and the results were plotted against time in Figure $2 \mathrm{~B}$. As expected the percent of oxy $\mathrm{Hb}$ decreased over time, with $\mathrm{Hb}$ proceeding from $100 \%$ oxy $\mathrm{Hb}$ to $6.46 \%$ oxy $\mathrm{Hb}$.

\section{Comparison to the TCS Hemox Analyzer}

We compared the effects of allosteric modifiers on $\mathrm{Hb}-\mathrm{O}_{2}$ affinity in the TCS Hemox Analyzer and in the ODA. Figure 3A shows representative OECs of GBT $1118,{ }^{7}$ an analog of voxelotor, ${ }^{5,13}$ and phytic acid, ${ }^{17}$ a potent analog of 2,3-DPG, in comparison to $\mathrm{Hb}$ alone. GBT1118 has a left-shifted OEC and a lower p50 value while phytic acid has a rightshifted OEC and a higher p50 in comparison to $\mathrm{Hb}$ control (Table 1). Figure 3B shows the \%oxy $\mathrm{Hb}$ values over time for phytic acid and GBT1118 in the ODA. The data indicate that GBT1118 delays the transition from oxy $\mathrm{Hb}$ to deoxy $\mathrm{Hb}$ while phytic acid accelerates the transition. Based upon data from Figure 3B, left-shifting compounds seem to be best evaluated at longer deoxygenation times (108 minutes). On the other hand, right-shifting compounds (such as phytic acid) seem to be better differentiated at earlier times (30 minutes).

Based upon the changes observed with reference compounds, a large-scale comparison was performed to establish how left-shifters (compounds with left-shifted OECs compared to the $\mathrm{Hb}$ control) and right-shifters (compounds with right-shifted OECs compared to the $\mathrm{Hb}$ control) behaved in the TCS Hemox Analyzer and ODA. Using a subset of GBT Hb modifiers, a correlation was established between the $\Delta \mathrm{p} 50 \mathrm{~s}\left(\mathrm{p} 50_{\text {sample }}-\mathrm{p} 50_{\text {control }}\right)$ generated on the TCS Hemox Analyzer and the $\Delta \%$ oxy $\mathrm{Hb}\left(\%\right.$ oxy $\mathrm{Hb}_{\text {sample }}-\%$ oxy $\left.\mathrm{Hb}_{\text {control }}\right)$ obtained in the ODA. Figure $3 \mathrm{C}$ and D shows the correlations between $\Delta \mathrm{p} 50$ and $\Delta \%$ oxy $\mathrm{Hb}$ for various compounds. A correlation $\left(R^{2}\right)$ of 0.70 was obtained for both left-shifter (with \%oxy $\mathrm{Hb}$ measured at 108 minutes) and right-shifter compounds (with \%oxy $\mathrm{Hb}$ measured at 30 minutes) indicating that this is a relevant primary screening assay to filter out inactive compounds as well as to rank compounds based on $\mathrm{Hb}$ modifying potency. The other major advantage is the small amount of compound required - reducing compound
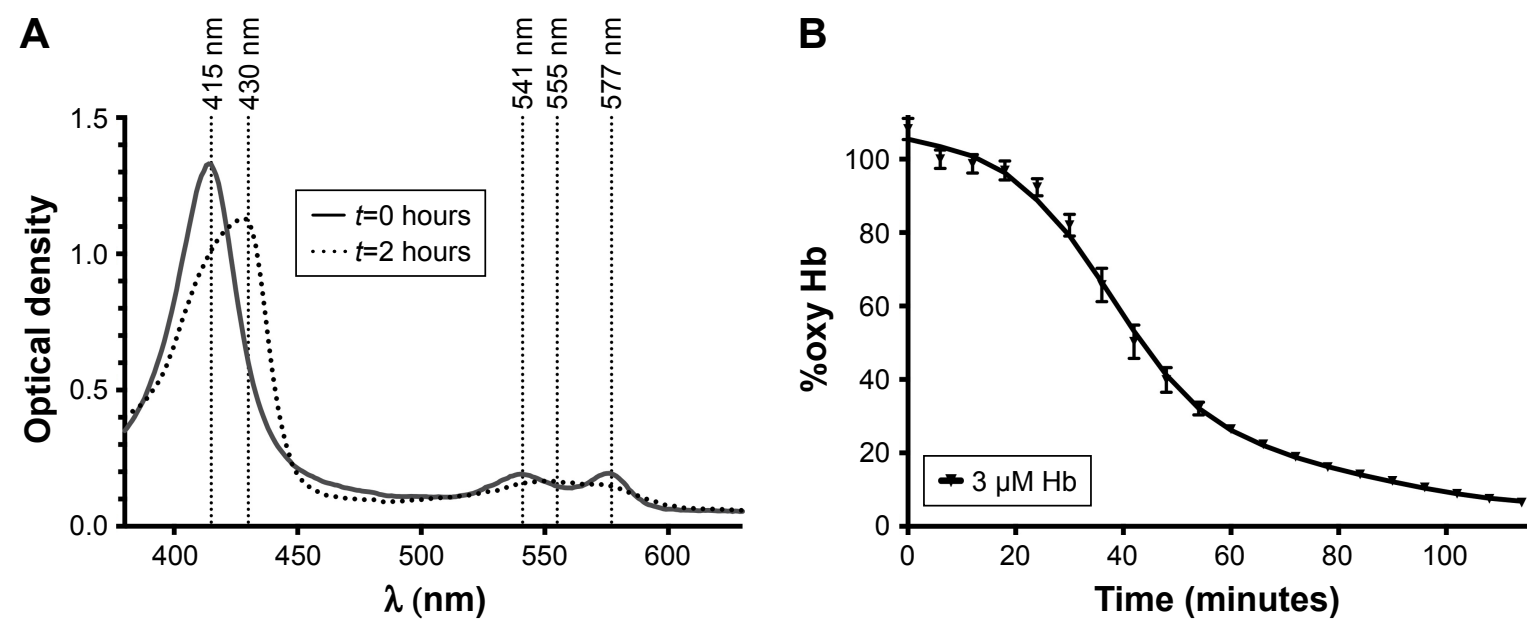

Figure 2 Spectra of $\mathrm{Hb}$ over 2 hours of deoxygenation.

Notes: The representative spectrum in (A) indicates that at $t=0$ hours fully oxygenated $\mathrm{Hb}$ exists within the well (peaks at $4 \mathrm{I} 5,54 \mathrm{I}$, and $577 \mathrm{~nm}$ ). After 2 hours, the peak shifts toward 430 and $555 \mathrm{~nm}$ (respectively), indicating that Hb shifts to a significantly deoxygenated state (6.46 \%oxy Hb). (B) Using Excel's LINEST function, the 20 spectra collected over the 2-hour period of deoxygenation were analyzed and plotted against time to quantitate the level of $\mathrm{Hb}$ deoxygenation (data collected in triplicate). All experiments were conducted in phosphate buffer.

Abbreviations: $\mathrm{Hb}$, hemoglobin; oxy $\mathrm{Hb}$, oxygenated $\mathrm{Hb}$. 

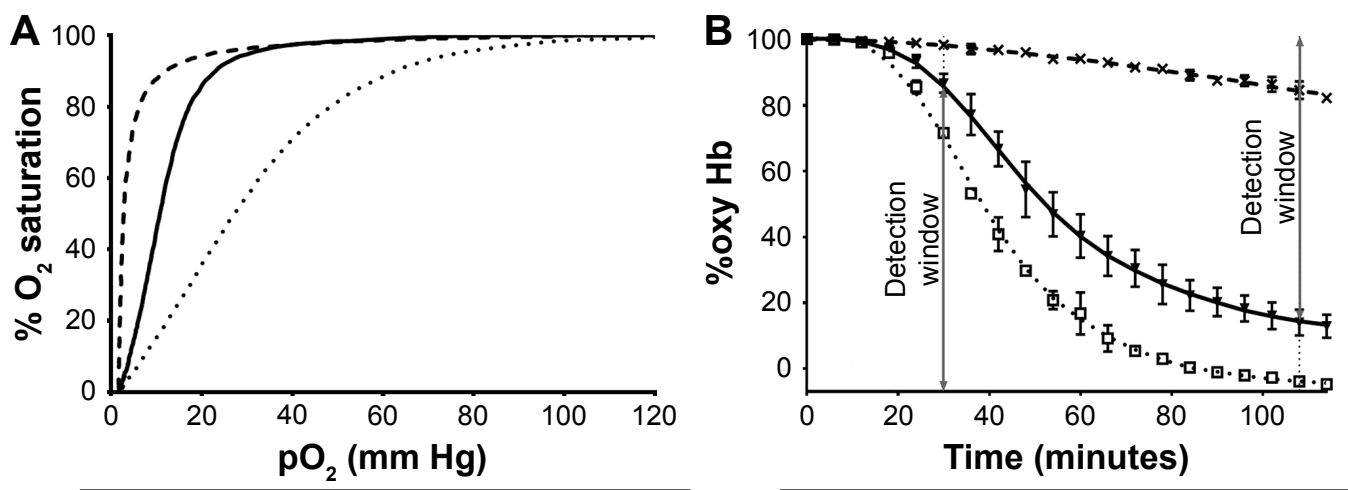

-. Phytic acid $(100 \mu \mathrm{M})=-$ GBT1118 $(30 \mu \mathrm{M})$
- Hb control

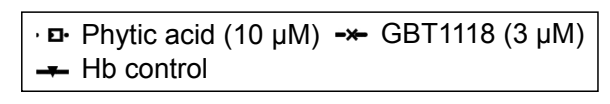

C
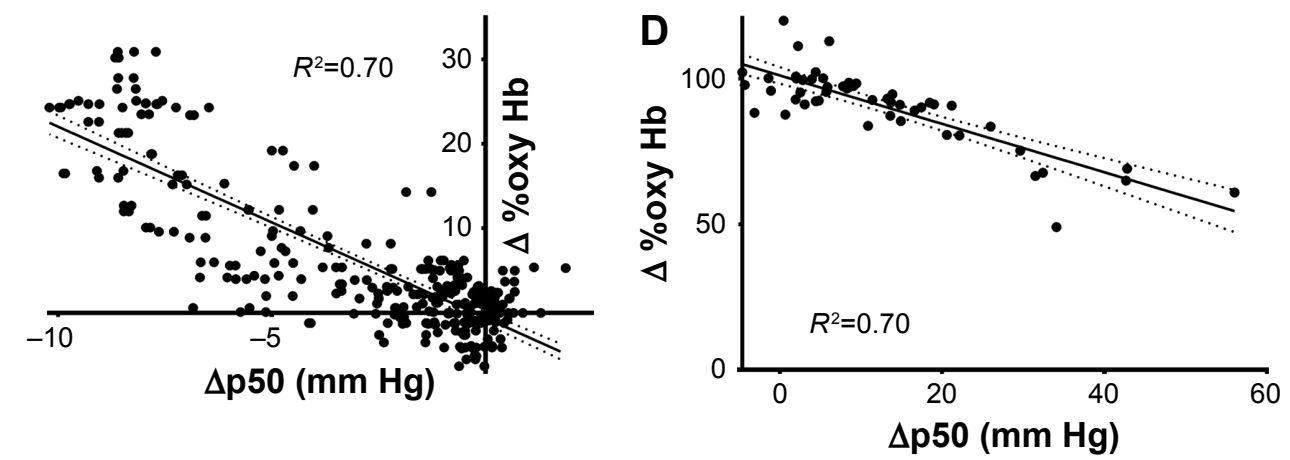

Figure 3 OECs and oxygen dissociation assay are highly correlated.

Notes: (A) Representative OECs of GBTIII 8 (at $30 \mu \mathrm{M}$ ) and phytic acid (at $100 \mu$ M) from samples run in the TCS Hemox Analyzer (25 $\mu$ M Hb). GBTII I 8 shows a leftward curve shift and lowers the $\mathrm{p} 50$. Phytic acid exhibits a rightward shift and higher p50. (B) The \%oxy Hb values over time for Hb (3 $\mu$ M), phytic acid, and GBTII I 8 in the ODA. The deoxygenation of $\mathrm{Hb}$ solution over time in the presence of GBTI I I8, which stabilizes the oxygenated state of $\mathrm{Hb}$, and phytic acid, which sustains the deoxygenated state of $\mathrm{Hb}$. All experiments in (A and B) were conducted in TES buffer. (C) Three hundred eleven compounds were run in the TCS Hemox Analyzer and ODA at I00 $\mu$ M in TES buffer and $10 \mu \mathrm{M}$ phosphate buffer, respectively. (D) Fifty-one compounds were tested in the TCS Hemox and ODA at 500 and $60 \mu \mathrm{M}$, respectively, in TES buffer.

Abbreviations: $\mathrm{Hb}$, hemoglobin; ODA, oxygen dissociation assay; OECs, oxygen equilibrium curves.

aggregation - compared to the greater quantity of compound needed to conduct the assay in a TCS Hemox Analyzer.

The robustness of the assay was determined with a variety of parameters. The intraplate variability is $3.0 \%-8.0 \%$ coefficient of variation (CV) for 30 minutes and $8.9 \%-13.7 \% \mathrm{CV}$ for 108 minutes. Similarly, the interplate variability is $3.1 \%-3.9 \%$ $\mathrm{CV}$ for 30 minutes and $19.4 \%-22.2 \% \mathrm{CV}$ for 108 minutes. The $Z^{\prime}$ for 30 minutes is 0.52 while the $Z^{\prime}$ for 108 minutes is 0.83 .

\section{Various uses for ODA \\ Dose-response curves}

Evaluating dose responses is a standard procedure in drug discovery to evaluate a compound potency. Dose responses

Table I p50 of purified $\mathrm{Hb}$ in the presence of $\mathrm{Hb}$ modifiers

\begin{tabular}{ll}
\hline Sample & p50 (N), mm Hg \\
\hline Hb control & $9.75 \pm 0.54(8)$ \\
GBTI I I8 $(30 \mu M)$ & $2.69 \pm 0.03(3)$ \\
Phytic acid $(100 \mu \mathrm{M})$ & $27.75 \pm 0.75(2)$ \\
\hline
\end{tabular}

Note: Data presented as mean \pm SD.

Abbreviations: $\mathrm{Hb}$, hemoglobin; $\mathrm{p} 50$, the partial pressure of $\mathrm{O}_{2}$ at which $\mathrm{Hb}$ is $50 \%$ saturated with $\mathrm{O}_{2}$. help determine the dose necessary to achieve the desired effect for a given ligand/receptor combination. However, in the $\mathrm{Hb}$ modifier field, standard dose responses in triplicate have been hindered by the limitation of the instrumentation. For example, in a TCS Hemox Analyzer, a seven-point dose response in triplicate for a single compound would take 3 days, while the same experiment can be completed in a single plate alongside three other $\mathrm{Hb}-\mathrm{O}_{2}$ affinity modifiers within 3.5 hours using the ODA. Figure 4 shows examples of ODA dose response of two compounds, GBT1118 (a left-shifter, Figure 4A) and phytic acid ${ }^{17}$ (a right-shifter, Figure 4B), previously described in the literature.

\section{$\mathrm{pH}$-dependent $\mathrm{Hb}-\mathrm{O}_{2}$ affinity}

A major property of $\mathrm{Hb}$ is its ability to deliver oxygen based upon the local tissue needs. ${ }^{2} \mathrm{Hb}$ achieves this through multiple means, one of which is the ability of $\mathrm{Hb}$ to change $\mathrm{Hb}-\mathrm{O}_{2}$ affinity based upon the hydrogen ion concentration, known as the Bohr effect. ${ }^{2}$ Metabolically active, or oxygenstarved tissues have a higher demand for oxygen, a buildup of $\mathrm{CO}_{2}$ and switch from aerobic metabolism to anaerobic 

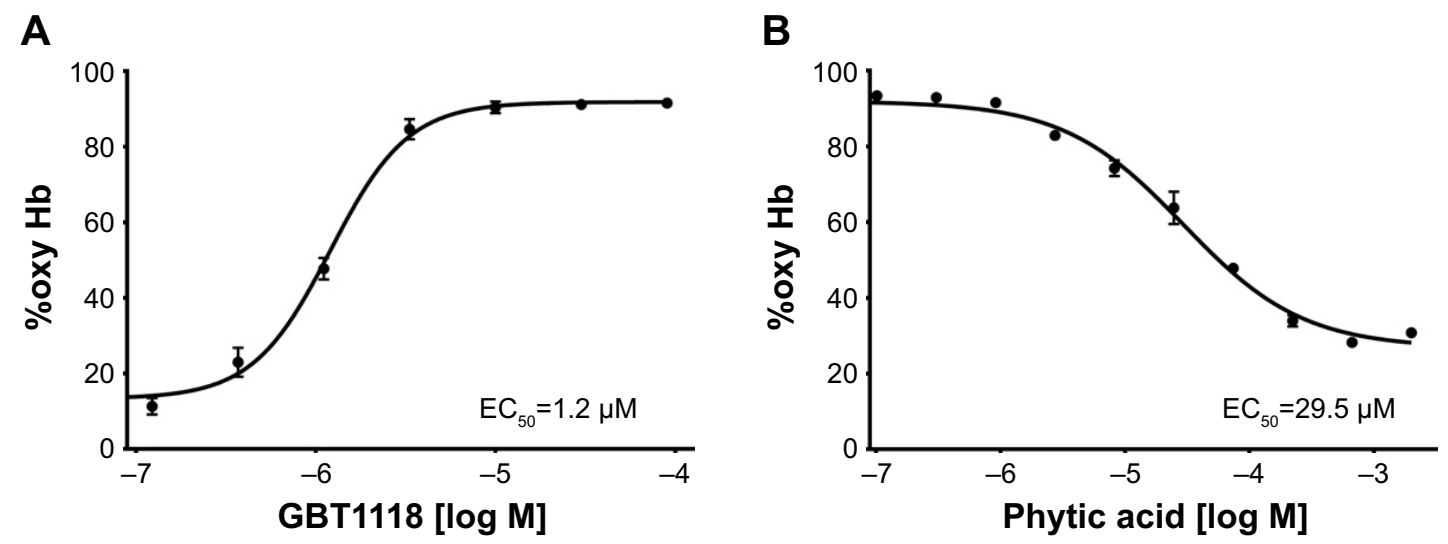

Figure 4 Dose responses obtained using the oxygen dissociation assay.

Notes: (A) Dose response of GBTIII8, a "left-shifting" Hb modifier collected in triplicate using the ODA; \%oxy Hb values were collected at 108 minutes. (B) Dose response of phytic acid, a "right-shifting" $\mathrm{Hb}$ modifier using the ODA; \%oxy Hb values were collected at 30 minutes. All experiments were conducted in triplicate in TES buffer. Data are represented as mean \pm SD.

Abbreviations: $\mathrm{Hb}$, hemoglobin; ODA, oxygen dissociation assay; oxy $\mathrm{Hb}$, oxygenated $\mathrm{Hb}$; $\mathrm{EC}_{50}$, half maximal effective concentration.

metabolism, which increases the lactic acid concentration. The combination of the increasing $\mathrm{CO}_{2}$ and increasing lactic acid levels lowers the $\mathrm{pH}$ of the oxygen-starved tissues. Due to the Bohr effect, under conditions of lowered $\mathrm{pH}$, the $\mathrm{Hb}-\mathrm{O}_{2}$ affinity decreases, allowing for $\mathrm{Hb}$ to deliver more oxygen. ${ }^{18}$ As this is a key property of $\mathrm{Hb}-\mathrm{O}_{2}$ affinity modifiers, it was necessary to ensure that the ODA could capture the effect of $\mathrm{pH}$. Figure $5 \mathrm{~A}$ shows how $\mathrm{Hb}$ behaves over time in the ODA. As expected, $\mathrm{Hb}$ at $\mathrm{pH} 6.8$ is more rapidly deoxygenated than $\mathrm{Hb}$ at $\mathrm{pH} 7.4$, while $\mathrm{Hb}$ at $\mathrm{pH} 8$ sustains the oxy $\mathrm{Hb}$ state for a longer time frame. Figure 5B shows that GBT1118 maintains a similar \% of $\mathrm{Hb}$ in the oxy $\mathrm{Hb}$ state regardless
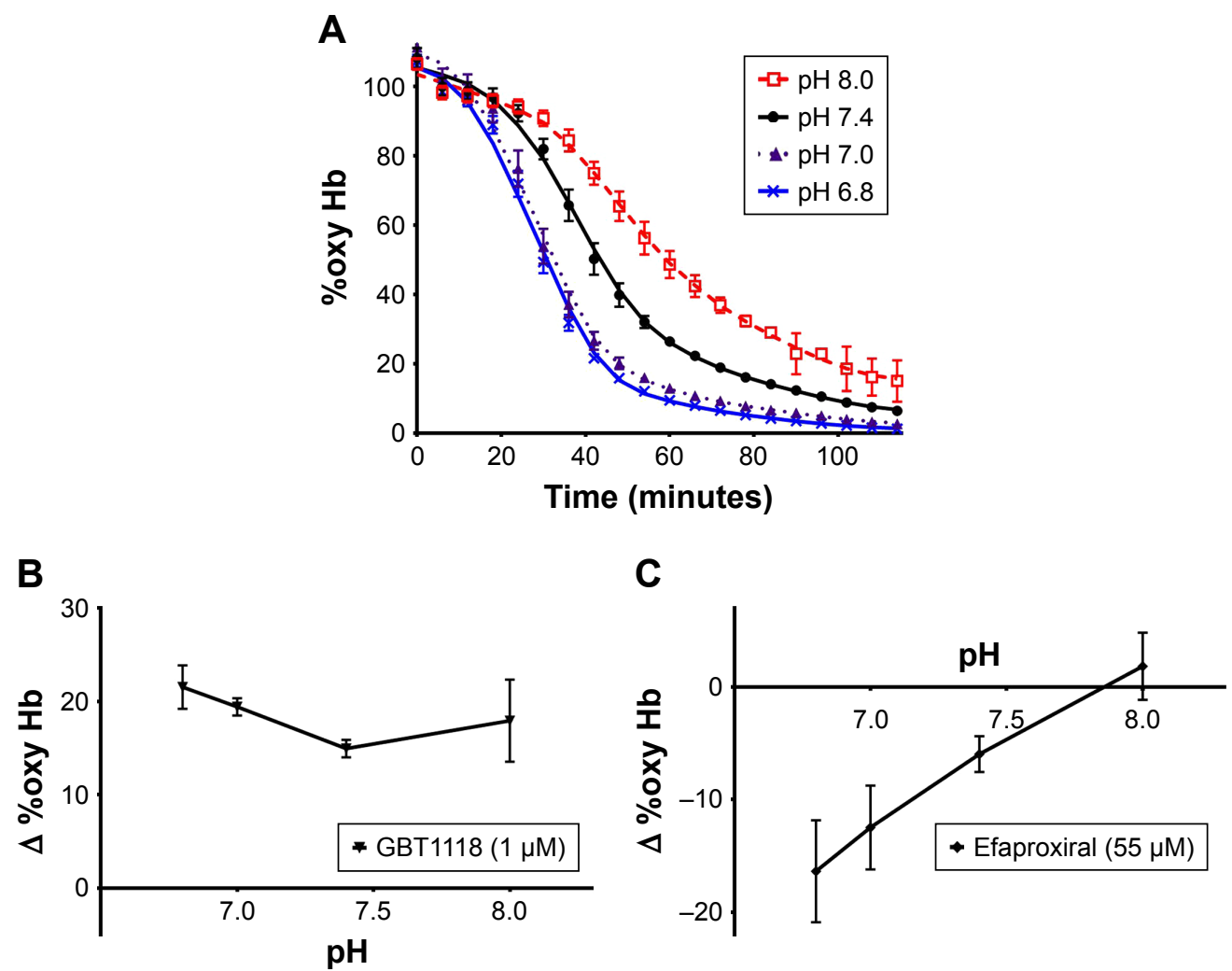

Figure $5 \mathrm{Hb}-\mathrm{O}_{2}$ affinity is $\mathrm{pH}$ dependent.

Notes: (A) \%oxy Hb measured for $3 \mu \mathrm{M} \mathrm{Hb}$ tetramer at various $\mathrm{pH}$. As the $\mathrm{pH}$ of the buffer is decreased, a decrease in \%oxy $\mathrm{Hb}$ can be observed for any given time. The assay was conducted using phosphate buffer. (B) Analysis of GBTIII 8 at various $\mathrm{pHs}$ using the ODA. The $\Delta \%$ oxy $\mathrm{Hb}$ values were collected at 108 minutes. Experiments were conducted in triplicate in TES buffer. Data are represented as mean \pm SD. (C) Analysis of efaproxiral using the ODA. The $\Delta \%$ oxy $\mathrm{Hb}$ values were collected at 30 minutes. Experiments were conducted in triplicate in TES buffer. Data are represented as mean \pm SD.

Abbreviations: $\mathrm{Hb}$, hemoglobin; ODA, oxygen dissociation assay; oxy $\mathrm{Hb}$, oxygenated $\mathrm{Hb}$. 
of $\mathrm{pH}$, which is ideal for a compound designed to maintain a portion of $\mathrm{Hb}$ in the oxy $\mathrm{Hb}$ state in order to delay $\mathrm{HbS}$ polymerization and prevent sickling in SCD patients. ${ }^{13}$ Even though there was a response to the changing $\mathrm{pH}$, GBT1118 is able to maintain an approximately equal portion of $\mathrm{Hb}$ in the oxy $\mathrm{Hb}$ state, thereby ensuring that it will be effective at delaying $\mathrm{HbS}$ polymerization even in metabolically active tissues. On the other hand, Figure 5C shows that efaproxiral is more effective at deoxygenating $\mathrm{Hb}$ at lower $\mathrm{pHs}$, which is ideal for a compound that was designed to deliver more oxygen to hypoxic tissues. ${ }^{19}$ By having a small decrease in $\mathrm{Hb}-\mathrm{O}_{2}$ affinity at $\mathrm{pH} 7.4$, it ensures that $\mathrm{Hb}$ is still able to load oxygen in the lungs, while at $\mathrm{pH} 6.8$ in the presence of efaproxiral, the $\mathrm{Hb}-\mathrm{O}_{2}$ affinity is significantly decreased, thereby allowing for maximal unloading of oxygen. Table 2 shows the p50s measured for GBT1118 and efaproxiral at $\mathrm{pH} 6.8$ and 8.0. GBT1118 shows a small change $(\Delta \mathrm{p} 50)$ between the two $\mathrm{pHs}(-0.5$ to $-3.2 \mathrm{~mm} \mathrm{Hg}$ ), while efaproxiral shows a significant increase in $\Delta \mathrm{p} 50$ going from $\mathrm{pH} 8.0$ to pH 6.8 ( 5 to $22.7 \mathrm{~mm} \mathrm{Hg}$ ). These data corroborate well with what is observed in the ODA.

\section{Albumin vs $\mathrm{Hb}$ binding}

Finally, the ODA can be used to screen and select $\mathrm{Hb}$ modifiers with appropriate protein binding profiles. Depending on the desired output, the compound can be preincubated with either $\mathrm{Hb}$ or albumin first and then the second protein added. In Figure 6, the samples were preincubated with albumin for 1 hour and then $\mathrm{Hb}$ was added to the system followed by a subsequent 1 -hour incubation. The data in Figure 6 indicate that compared to tucaresol, a compound known to have high affinity for plasma proteins, ${ }^{20}$ voxelotor has a tenfold greater affinity for $\mathrm{Hb}$ over albumin. This is demonstrated by the ability of voxelotor to maintain a higher proportion of oxy $\mathrm{Hb}$ in the presence of HSA $\left(\mathrm{HSA} \mathrm{IC}_{50}=10 \mu \mathrm{M}\right.$ ) when compared to tucaresol (HSA $\left.\mathrm{IC}_{50}=1.4 \mu \mathrm{M}\right)$. The preferential voxelotor binding to $\mathrm{Hb}$ compared to tucaresol likely contributed to the greater RBC:plasma partitioning of voxelotor with less voxelotor remaining in the plasma compartment upon oral dosing

Table 2 The effect of $\mathrm{pH}$ on the $\mathrm{p} 50 \mathrm{~s}$ of purified $\mathrm{Hb}$ in the presence of $\mathrm{Hb}$ modifiers

\begin{tabular}{lll}
\hline & \multicolumn{2}{c}{$\mathbf{p H}$} \\
\cline { 2 - 3 } & $\mathbf{6 . 8}$ & $\mathbf{8 . 0}$ \\
\hline Control & 14.6 & 5 \\
GBTIII8 $(10 \mu \mathrm{M})$ & 11.4 & 4.5 \\
Efaproxiral $(500 \mu \mathrm{M})$ & 37.3 & 10 \\
\hline
\end{tabular}

Abbreviation: $\mathrm{Hb}$, hemoglobin.

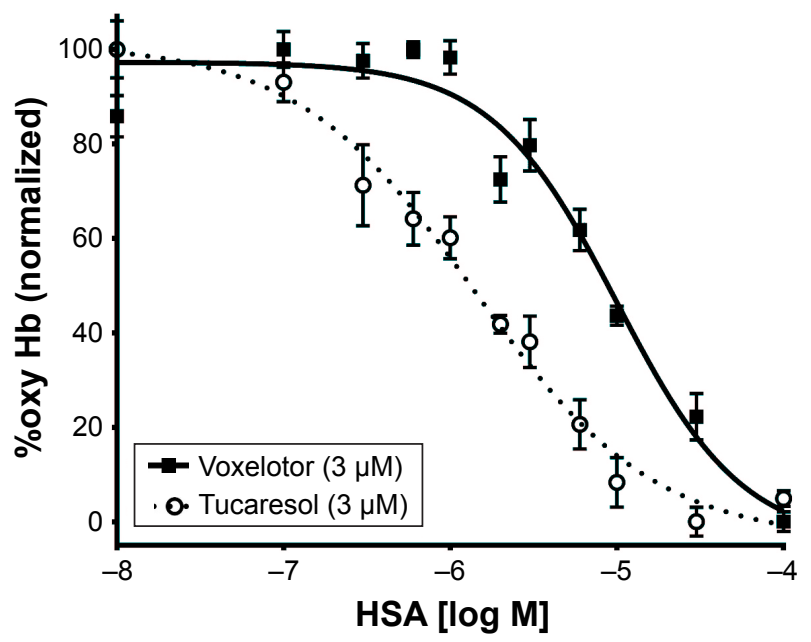

Figure 6 Albumin vs $\mathrm{Hb}$ binding.

Note: The activity of both voxelotor $(3 \mu \mathrm{M})$ and tucaresol $(3 \mu \mathrm{M})$ diminishes with increasing concentrations of HSA.

Abbreviations: HSA, human serum albumin; Hb, hemoglobin; oxy Hb, oxygenated $\mathrm{Hb}$.

to humans (NCT02285088). ${ }^{20-22}$ This method can clarify the relative affinities of a given compound for $\mathrm{Hb}$ vs HSA, or the nonspecific binding contributions in whole blood.

\section{Discussion}

The use of $\mathrm{Hb}-\mathrm{O}_{2}$ affinity modifiers has been investigated in depth to target diseases, such as $\mathrm{SCD}, 5,6$ pulmonary diseases with associated hypoxemia (eg, IPF), ${ }^{7}$ tumor hypoxia, ${ }^{23,24}$ wound healing, ${ }^{1}$ and ischemia. ${ }^{1}$ However, optimizing such modifiers through SAR for drug discovery has proven difficult because of the limitations of available analytical methodologies. The TCS Hemox Analyzer is a well-established instrument convenient for clinical samples. However, it requires a significant amount of time and compound in order to conduct SAR studies. In this paper, we present the ODA assay - a modular, medium-throughput assay conducive to simultaneously screen $\mathrm{Hb}-\mathrm{O}_{2}$ affinity modifiers in a variety of conditions in small volumes. These conditions include, but are not limited to: $\mathrm{pH}$, buffers, and coincubation with proteins such as HSA. As shown in the voxelotor vs tucaresol experiment, measuring HSA effects could prove predictive of pharmacokinetic parameters or RBC:plasma partitioning. This predictive power was confirmed by the data from the clinical trials of voxelotor and tucaresol. ${ }^{20-22}$ In the Phase I/ IIa trial, voxelotor (NCT02285088) was shown to partition preferentially into the $\mathrm{RBC}$ compartment in relation to plasma (60-90:1), which indicates a high affinity and specificity for $\mathrm{Hb}^{21}$ To the contrary, tucaresol, which exhibited a higher HSA IC $_{50}$ (Figure 6), had a much poorer RBC:plasma partitioning $(10-13: 1){ }^{20,22}$ 
In the ODA, we screened over 500 compounds, a combination of left and right-shifters, and showed that this assay can be used to rapidly screen compounds and determine half maximal effective concentration $\left(\mathrm{EC}_{50}\right)$. We also showed the ability of the ODA to be adapted to characterize compounds with respect to $\mathrm{pH}$ and albumin binding sensitivity. Though the ODA is ideal for primary screening, the TCS Hemox Analyzer would still be necessary for a detailed characterization of the $\mathrm{Hb}-\mathrm{O}_{2}$ affinity as the ODA does not measure the partial pressure of $\mathrm{O}_{2}$ throughout the assay.

One of the major challenges in developing ODA was determining the optimal $\mathrm{Hb}$ concentration. Many screening assays use protein concentrations within the pico- to nano molar range in an effort to minimize compound needs and compound solubility issues. However, at such concentrations, the $\mathrm{Hb}$ tetramer dissociates to dimers making lower $\mathrm{Hb}$ concentrations a non-optimal choice for a screening campaign. Conversely, as the TCS Hemox Analyzer uses a Hb tetramer concentration of $25 \mu \mathrm{M}$, compounds would regularly be above the solubility limit preventing accurate quantitation of the $\mathrm{Hb}-\mathrm{O}_{2}$ affinity changes. Based upon the literature references in similar buffer systems, a concentration of $3 \mu \mathrm{M}$ tetramer became an ideal choice, with $\mathrm{Hb}$ levels above the tetramer:dimer equilibrium. ${ }^{15,16}$ This will still allow for a tenfold decrease in compound concentration (compared to the TCS Hemox), which would ensure that most compounds are below their solubility limit. The secondary benefit gained by decreasing the $\mathrm{Hb}$ concentration while simultaneously reducing the volume (TCS Hemox cuvette uses $5 \mathrm{~mL}$, while ODA uses $160 \mu \mathrm{L} /$ well) ensured a 300-fold total reduction in compound needed for primary screening and SAR studies. Though $3 \mu \mathrm{M} \mathrm{Hb}$ tetramer concentrations could occasionally lead to increases in $\mathrm{Hb}$ dimers under different $\mathrm{pHs}$ or in the presence of tetramer-destabilizing compounds, a secondary screen using the TCS Hemox will allow confirmation of the ODA hits. On the other hand, if compound solubility is not a concern, the assay and data analysis can be adapted to 10 or $25 \mu \mathrm{M} \mathrm{Hb}$ tetramer concentrations.

As detailed above, the ODA examines the ability of compounds to modulate the release of $\mathrm{O}_{2}$ from $\mathrm{Hb}$ under various conditions. This is exemplified by the ODA experiments at various $\mathrm{pHs}$. To conduct a similar experiment with the Hemox Analyzer, the data collection alone would take 2 weeks, which would add day-to-day variability to the data. In addition, such an experiment would require a great amount of compound (1.3 mL for the TCS Hemox Analyser vs $4 \mu \mathrm{L}$ for the ODA from a $10 \mathrm{mM}$ stock). On the other hand, the ODA can complete such a comparison in triplicate within
3.5 hours in the same day eliminating day-to-day variability, and with $<1 \%$ of the compound needed.

Although Nakagawa et $\mathrm{al}^{12}$ have reported a similar assay, the ODA goes a step further by decreasing the Hb concentration to $3 \mu \mathrm{M}$ (vs $10 \mu \mathrm{M}$ ) and the assay time to 2 hours (vs 14 hours) and shows a significant correlation with the TCS Hemox Analyzer for both left-shifters and right-shifters. Finally, one of the strengths of the ODA is its adaptability, allowing researchers to use the assay for both screening and characterizing $\mathrm{Hb}$ modifiers. As this article shows, the ODA allowed GBT1118 and efaproxiral to be characterized at a variety of $\mathrm{pHs}$ to elucidate the role of $\mathrm{pH}$ on $\mathrm{Hb}-\mathrm{O}_{2}$ affinity modification. Similarly, the albumin vs $\mathrm{Hb}$ assay can aid in rank-ordering compounds for in vivo studies.

\section{Conclusion}

This novel assay (ODA) is simple, reproducible, and adaptable and requires no special reagents. It has been evaluated against the TCS Hemox Analyzer with over 500 compounds and displayed a correlation of 0.7 for both right and leftshifting $\mathrm{Hb}$ modifiers. In addition, the ODA platform has been used to characterize various compounds ${ }^{5}$ including those discussed in this article: GBT1118, phytic acid, voxelotor, tucaresol, and efaproxiral. The equipment cost is comparable to a single TCS Hemox and can easily be used for SAR in a screening setting to identify novel $\mathrm{Hb}-\mathrm{O}_{2}$ affinity modifiers. The use of the ODA will improve the throughput and allow for a rapid development of novel $\mathrm{Hb}-\mathrm{O}_{2}$ affinity modifiers, which will be beneficial to a variety of diseases.

\section{Disclosure}

Mira P Patel, Vincent Siu, Abel Silva-Garcia, Qing Xu, Zhe Li, and Donna Oksenberg are employees, and shareholders, of Global Blood Therapeutics, Inc. The authors report no other conflicts of interest in this work.

\section{References}

1. Safo MK, Ahmed MH, Ghatge MS, Boyiri T. Hemoglobin-ligand binding: understanding $\mathrm{Hb}$ function and allostery on atomic level. Biochim Biophys Acta. 2011;1814:797-809.

2. Mairbäurl H, Weber RE. Oxygen transport by hemoglobin. Compr Physiol. 2012;2:1463-1489.

3. Duarte CD, Greferath R, Nicolau C, Lehn JM. Myo-inositol trispyrophosphate: a novel allosteric effector of hemoglobin with high permeation selectivity across the red blood cell plasma membrane. ChemBioChem. 2010;11:2543-2548.

4. Sahu SC, Simplaceanu V, Gong Q, et al. Insights into the solution structure of human deoxyhemoglobin in the absence and presence of an allosteric effector. Biochemistry. 2007;46:9973-9980.

5. Oksenberg D, Dufu K, Patel MP, et al. GBT440 Increases haemoglobin oxygen affinity, reduces sickling and prolongs $\mathrm{RBC}$ half-life in a murine model of sickle cell disease. Br J Haematol. 2016;175:141-153. 
6. Safo MK, Kato GJ. Therapeutic strategies to alter the oxygen affinity of sickle hemoglobin. Hematol Oncol Clin North Am. 2014;28: 217-231.

7. Geng X, Dufu K, Hutchaleelaha A, et al. Increased hemoglobin-oxygen affinity ameliorates bleomycin-induced hypoxemia and pulmonary fibrosis. Physiol Rep. 2016;4(17):e12965.

8. Dufu K, Yalcin O, Ao-ieong ES, et al. GBT1118, a potent allosteric modifier of hemoglobin oxygen affinity increases tolerance to severe hypoxia in mice. Am J Physiol - Hear Circ Physiol. 2017;313(2):H381-H391.

9. Amorino GP, Lee H, Holburn GE, et al. Enhancement of tumor oxygenation and radiation response by the allosteric effector of hemoglobin, RSR13. Radiat Res. 2001;156:294-300.

10. Kister J, Wajcman H. Oxygen equilibrium measurements of human red blood cells. Methods Mol Med. 2003;82:49-64.

11. Guarnone R, Centenara E, Barosi G. Performance characteristics of hemox-analyzer for assessment of the hemoglobin dissociation curve. Haematologica. 1995;80:426.

12. Nakagawa A, Lui FE, Wassaf D, et al. Identification of a small molecule that increases hemoglobin oxygen affinity and reduces SS erythrocyte sickling. ACS Chem Biol. 2014;9(10):2318-2325.

13. Metcalf B, Chuang C, Dufu K, et al. Discovery of GBT440, an orally bioavailable R state stabilizer of sickle cell hemoglobin. ACS Med Chem Lett. 2017;8(3):321-326.

14. Goldberg MA, Husson MA, Bunn HF. Participation of hemoglobins A and F in polymerization of aickle hemoglobin. J Biol Chem. 1977; 252:3414-3421.

15. Arisaka F, Nagai Y, Nagai M. Dimer-tetramer association equilibria of human adult hemoglobin and its mutants as observed by analytical ultracentrifugation. Methods. 2011;54:175-180.

16. Griffon N, Baudin V, Dieryck W, et al. Tetramer-dimer equilibrium of oxyhemoglobin mutants determined from auto-oxidation rates. Protein Sci. 1998;7:673-680.
17. Fylaktakidou KC, Lehn JM, Greferath R, Nicolau C. Inositol tripyrophosphate: a new membrane permeant allosteric effector of haemoglobin. Bioorg Med Chem Lett. 2005;15:1605-1608.

18. Jensen FB. Red blood cell $\mathrm{pH}$, the bohr effect, and other oxygenationlinked phenomena in blood $\mathrm{O} 2$ and $\mathrm{CO}_{2}$ transport. Acta Physiol Scand. 2004;182:215-227.

19. Kavanagh BD, Khandelwal SR, Schmidt-Ullrich RK, et al. A Phase I study of RSR13, a radiation-enhancing hemoglobin modifier: tolerance of repeated intravenous doses and correlation of pharmacokinetics with pharmacodynamics. Int J Radiat Oncol Biol Phys. 2001;49: 1133-1139.

20. Rolan PE, Parker JE, Gray SJ, et al. The pharmacokinetics, tolerability and pharmacodynamics of tucaresol (589C80; 4[2-Formyl-3Hydroxyphenoxymethyl] benzoic acid), a potential anti-sickling agent, following oral administration to healthy subjects. Br J Clin Pharmacol. 1993;35:419-425.

21. Lehrer-Graiwer J, Howard J, Hemmaway CJ, et al. GBT440, a potent anti-sickling hemoglobin modifier reduces hemolysis, improves anemia and nearly eliminates sickle cells in peripheral blood of patients with sickle cell disease. Blood. 2015;126:542.

22. Rolan PE, Mercer AJ, Wootton R, Posner J. Pharmacokinetics and pharmacodynamics of tucaresol, an antisickling agent, in healthy volunteers. Br J Clin Pharmacol. 1995;39:375-380.

23. Aprahamian M, Bour G, Akladios CY, et al. Myo-InositolTrisPyroPhosphate treatment leads to HIF-1 $\alpha$ suppression and eradication of early hepatoma tumors in rats. ChemBioChem. 2011;12:777-783.

24. Raykov Z, Grekova SP, Bour G, et al. Myo-inositol trispyrophosphatemediated hypoxia reversion controls pancreatic cancer in rodents and enhances gemcitabine efficacy. Int J Cancer. 2014;134:2572-2582.
Drug Design, Development and Therapy

\section{Publish your work in this journal}

Drug Design, Development and Therapy is an international, peerreviewed open-access journal that spans the spectrum of drug design and development through to clinical applications. Clinical outcomes, patient safety, and programs for the development and effective, safe, and sustained use of medicines are the features of the journal, which

\section{Dovepress}

has also been accepted for indexing on PubMed Central. The manuscript management system is completely online and includes a very quick and fair peer-review system, which is all easy to use. Visit http://www.dovepress.com/testimonials.php to read real quotes from published authors.

Submit your manuscript here: http://www.dovepress.com/drug-design-development-and-therapy-journal 